PROCEEDINGS OF THE

AMERICAN MATHEMATICAL SOCIETY

Volume 139, Number 3, March 2011, Pages 931-942

S 0002-9939(2010)10701-3

Article electronically published on November 1, 2010

\title{
BILATERAL $q$-WATSON AND $q$-WHIPPLE SUMS
}

\author{
WENCHANG CHU AND CHENYING WANG
}

(Communicated by Walter Van Assche)

\begin{abstract}
Watson and Whipple (1925) discovered two summation formulae for a ${ }_{3} F_{2}(1)$-series. Their terminating $q$-analogues were found by Andrews (1976) and Jain (1981). As a common extension of these results, we prove a general bilateral series identity, which may also be considered as a full $q$ analogue of the ${ }_{3} H_{3}$-series identity due to M. Jackson (1949). This will be accomplished by means of the modified Abel lemma on summation by parts.
\end{abstract}

For two indeterminates $x$ and $q$, the shifted-factorial of $x$ with base $q$ is defined by

$$
(x ; q)_{0}=1 \quad \text { and } \quad(x ; q)_{n}=(1-x)(1-q x) \cdots\left(1-q^{n-1} x\right) \quad \text { for } \quad n \in \mathbb{N} .
$$

When $|q|<1$, we have two well-defined infinite products

$$
(x ; q)_{\infty}=\prod_{k=0}^{\infty}\left(1-q^{k} x\right) \quad \text { and } \quad(x ; q)_{n}=(x ; q)_{\infty} /\left(q^{n} x ; q\right)_{\infty} .
$$

The product and fraction of shifted factorials are abbreviated respectively to

$$
\begin{aligned}
{[\alpha, \beta, \cdots, \gamma ; q]_{n} } & =(\alpha ; q)_{n}(\beta ; q)_{n} \cdots(\gamma ; q)_{n} \\
{\left[\begin{array}{c}
\alpha, \beta, \cdots, \gamma \\
A, B, \cdots, C
\end{array} \mid q\right]_{n} } & =\frac{(\alpha ; q)_{n}(\beta ; q)_{n} \cdots(\gamma ; q)_{n}}{(A ; q)_{n}(B ; q)_{n} \cdots(C ; q)_{n}} .
\end{aligned}
$$

Following Gasper-Rahman [5], the unilateral and bilateral basic hypergeometric series are defined by

$$
\begin{aligned}
{ }_{1+r} \phi_{s}\left[\begin{array}{c}
a_{0}, a_{1}, \cdots, a_{r} \\
b_{1}, \cdots, b_{s}
\end{array} \mid q ; z\right] & =\sum_{n=0}^{\infty}\left\{(-1)^{n} q^{\left(\begin{array}{c}
n \\
2
\end{array}\right)}\right\}^{s-r}\left[\begin{array}{c}
a_{0}, a_{1}, \cdots, a_{r} \\
q, b_{1}, \cdots, b_{s}
\end{array} \mid q\right]_{n} z^{n}, \\
r & \psi_{s}\left[\begin{array}{c}
a_{1}, a_{2}, \cdots, a_{r} \\
b_{1}, b_{2}, \cdots, b_{s}
\end{array} \mid q ; z\right]=\sum_{n=-\infty}^{\infty}\left\{(-1)^{n} q^{\left(\begin{array}{c}
n \\
2
\end{array}\right)}\right\}^{s-r}\left[\begin{array}{c}
a_{1}, a_{2}, \cdots, a_{r} \\
b_{1}, b_{2}, \cdots, b_{s}
\end{array} \mid q\right]_{n} z^{n},
\end{aligned}
$$

where the base $q$ will be restricted to $|q|<1$ for nonterminating $q$-series.

Received by the editors February 22, 2010.

2010 Mathematics Subject Classification. Primary 33D15; Secondary 05A30.

Key words and phrases. Abel's lemma on summation by parts, $q$-Whipple sum, $q$-Watson sum.

The second author is the corresponding author.

(C)2010 American Mathematical Society Reverts to public domain 28 years from publication 
In 1925, Watson [8] and Whipple 9] respectively found the following two ${ }_{3} F_{2}(1)$ sums (cf. [2, SS3.3-3.4])

$$
\begin{aligned}
& { }_{3} F_{2}\left[\begin{array}{c}
a, b, c \\
\frac{1+a+b}{2}, 2 c
\end{array} \mid 1\right]=\frac{\Gamma\left(\frac{1}{2}\right) \Gamma\left(\frac{1}{2}+c\right) \Gamma\left(\frac{1+a+b}{2}\right) \Gamma\left(\frac{1-a-b}{2}+c\right)}{\Gamma\left(\frac{1+a}{2}\right) \Gamma\left(\frac{1+b}{2}\right) \Gamma\left(\frac{1-a}{2}+c\right) \Gamma\left(\frac{1-b}{2}+c\right)}, \\
& { }_{3} F_{2}\left[\begin{array}{c}
a, 1-a, c \\
b, 1+2 c-b
\end{array} \mid 1\right]=\frac{2^{1-2 c} \pi \Gamma(b) \Gamma(1+2 c-b)}{\Gamma\left(\frac{a+b}{2}\right) \Gamma\left(\frac{1+a-b+2 c}{2}\right) \Gamma\left(\frac{1-a+b}{2}\right) \Gamma\left(\frac{2-a-b+2 c}{2}\right)},
\end{aligned}
$$

where the convergence conditions for $[8$ and $[9]$ are respectively $\Re(a+b-2 c)<1$ and $\Re(c)>0$. After a half century, different $q$-analogues of the last two formulae were established respectively by Andrews [1] and Jain [7].

This short paper aims to prove a common extension of bilateral series for the $q$-Watson and $q$-Whipple sums, which provides also a full $q$-analogue of the ${ }_{3} \mathrm{H}_{3}$ series identity due to M. Jackson [6, 1949]. A few summation and transformation formulae will be presented as consequences.

\section{Main theorem And CONSEQuences}

Throughout this section, $a, b, c, d$ are assumed to be four indeterminates such that the nonterminating series containing them as parameters are well defined.

Theorem 1. Under the conditions $|a / b|<1$ and $|c / d|<1$, the following bilateral $q$-series transformation holds:

$$
\begin{aligned}
{ }_{4} \psi_{4}\left[\begin{array}{c}
a, c, \sqrt{q b d},-\sqrt{q b d} \\
q b, q d, \sqrt{q a c},-
\end{array}-\sqrt{q a c} \mid q ; q\right]=\left[\begin{array}{c}
a, 1 / d \\
q b, q / c
\end{array} \mid q\right]_{\infty}\left[\begin{array}{c}
q^{2}, q b c, q / b c, q^{2} b / c, a c / b d \\
q a c, a / b, a / d, c / d, q / b d
\end{array} \mid q^{2}\right]_{\infty} \\
+\frac{1 / d}{1-a / d}\left[\begin{array}{c}
a, c \\
q b, q d
\end{array} \mid q\right]_{\infty} \frac{\left(q b d ; q^{2}\right)_{\infty}}{\left(q a c ; q^{2}\right)_{\infty}} \sum_{k=0}^{\infty} \frac{\left(q^{2} b / c ; q^{2}\right)_{k}}{\left(q^{2} a / d ; q^{2}\right)_{k}}\left(\frac{c}{d}\right)^{k} \\
+\frac{a}{1-a / d}\left[\begin{array}{c}
1 / b, 1 / d \\
q / a, q / c
\end{array} \mid q\right]_{\infty} \frac{\left(q / a c ; q^{2}\right)_{\infty}}{\left(q / b d ; q^{2}\right)_{\infty}} \sum_{k=0}^{\infty} \frac{\left(q^{2} b / c ; q^{2}\right)_{k}}{\left(q^{2} a / d ; q^{2}\right)_{k}}\left(\frac{a}{b}\right)^{k} .
\end{aligned}
$$

As we shall show in Section 4, this theorem implies M. Jackson's identity of bilateral $_{3} \mathrm{H}_{3}$-series [6, Eq. 2.3]. Furthermore, this theorem contains the following four $q$-Watson and $q$-Whipple formulae as special cases, which were found by Andrews (1976) and Jain (1981) previously.

Firstly, letting $b=1, c=q^{-n}$ in the last theorem and then renaming the parameters by $a \rightarrow q^{1+n} a, d \rightarrow c / q$, we recover Andrews' terminating $q$-Watson formula.

Corollary 2 (Andrews' terminating ${ }_{4} \phi_{3}$-series identity [1]).

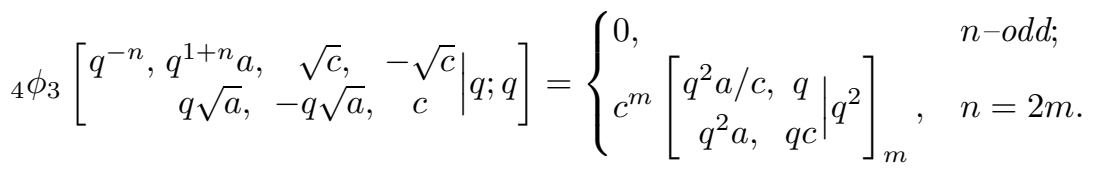

Instead, when $b \rightarrow 1$ and $d \rightarrow q^{-1-2 n}$, we get another $q$-Watson formula.

Corollary 3 (Jain's terminating ${ }_{4} \phi_{3}$-series identity [7, Eq. 3.17]).

$$
{ }_{4} \phi_{3}\left[\begin{array}{cccc}
a, c, & q^{-n}, & -q^{-n} \\
q^{-2 n}, & \sqrt{q a c}, & -\sqrt{q a c}
\end{array} \mid q ; q\right]=\left[\begin{array}{cc}
q a, & q c \\
q, & q a c
\end{array} \mid q^{2}\right]_{n} .
$$


Then letting $a \rightarrow q^{1+n}$ and $c \rightarrow q^{-n}$ yields Andrews' $q$-Whipple formula.

Corollary 4 (Andrews' terminating ${ }_{4} \phi_{3}$-series identity [1]).

$$
{ }_{4} \phi_{3}\left[\begin{array}{ccc}
q^{-n}, q^{1+n}, & \sqrt{q b d}, & -\sqrt{q b d} \\
-q, & q b, & q d
\end{array} \mid q ; q\right]=\frac{\left[q^{1-n} b, q^{1-n} d ; q^{2}\right]_{n}}{[q b, q d ; q]_{n}} q^{\left(\begin{array}{c}
n+1 \\
2
\end{array}\right)} .
$$

Finally, when $c \rightarrow q / a$ and $d \rightarrow q^{-1-2 n} / b$, we obtain another $q$-Whipple formula.

Corollary 5 (Jain's terminating ${ }_{4} \phi_{3}$-series identity [7, Eq. 3.19]).

$$
{ }_{4} \phi_{3}\left[\begin{array}{c}
a, q / a, q^{-n},-q^{-n} \\
-q, q b, q^{-2 n} / b
\end{array} \mid q ; q\right]=\frac{\left[q a b, q^{2} b / a ; q^{2}\right]_{n}}{(q b ; q)_{2 n}} .
$$

In addition, for $d=1$ in Theorem 1, we have the following surprising formula which transforms the balanced ${ }_{4} \phi_{3}$-series into a simpler one.

Corollary 6 (Nonterminating series transformation: $|c|<1$ ).

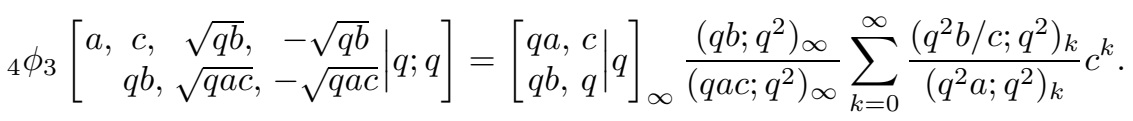

\section{Proof via Abel's lemma on summation by parts}

In order to prove Theorem 1] we shall utilize the modified Abel lemma on summation by parts [3, 4]. Given an arbitrary complex sequence $\left\{\tau_{k}\right\}$, define the backward and forward difference operators $\nabla$ and $\triangle$, respectively, by

$$
\nabla \tau_{k}=\tau_{k}-\tau_{k-1} \quad \text { and } \quad \Delta \tau_{k}=\tau_{k}-\tau_{k+1},
$$

where $\triangle$ differs from the usual operator $\Delta$ only in the minus sign.

Lemma 7 (Abel's lemma on summation by parts). Let $\left\{A_{k}\right\}$ and $\left\{B_{k}\right\}$ be two sequences such that the following two limits exist:

$$
[A B]_{+}:=\lim _{n \rightarrow+\infty} A_{n} B_{n+1} \text { and }[A B]_{-}:=\lim _{n \rightarrow-\infty} A_{n} B_{n+1} .
$$

Then the following transformation formula holds:

$$
\sum_{k=-\infty}^{+\infty} B_{k} \nabla A_{k}=[A B]_{+}-[A B]_{-}+\sum_{k=-\infty}^{+\infty} A_{k} \Delta B_{k}
$$

provided that one of the nonterminating series just displayed is convergent.

Proof. According to the definition of the backward difference, we have

$$
\sum_{k=-m}^{n} B_{k} \nabla A_{k}=\sum_{k=-m}^{n} B_{k}\left\{A_{k}-A_{k-1}\right\}=\sum_{k=-m}^{n} A_{k} B_{k}-\sum_{k=-m}^{n} A_{k-1} B_{k} .
$$

Replacing $k$ by $k+1$ for the last sum, we can reformulate the equation as follows:

$$
\begin{aligned}
\sum_{k=-m}^{n} B_{k} \nabla A_{k} & =A_{n} B_{n+1}-A_{-m-1} B_{-m}+\sum_{k=-m}^{n} A_{k}\left\{B_{k}-B_{k+1}\right\} \\
& =A_{n} B_{n+1}-A_{-m-1} B_{-m}+\sum_{k=-m}^{n} A_{k} \Delta B_{k} .
\end{aligned}
$$

Letting $m, n \rightarrow \infty$ leads to the equality stated in the modified Abel lemma. 
Now we are ready to prove Theorem 1 . First, define the $\Omega$-function by

$$
\Omega(a, b, c, d)=\sum_{k=-\infty}^{\infty}\left[\begin{array}{cc}
a, & c \\
q b, q d
\end{array} \mid q\right]_{k} \frac{\left(q b d ; q^{2}\right)_{k}}{\left(q a c ; q^{2}\right)_{k}} q^{k}
$$

For the two sequences given by

$$
A_{k}=\frac{(q a ; q)_{k}\left(q^{3} b d ; q^{2}\right)_{k}}{(q d ; q)_{k}\left(q^{3} a b ; q^{2}\right)_{k}} \quad \text { and } \quad B_{k}=\frac{(c ; q)_{k}\left(q^{3} a b ; q^{2}\right)_{k}}{\left(q^{2} b ; q\right)_{k}\left(q a c ; q^{2}\right)_{k}}
$$

it is almost trivial to compute the relations

$$
\begin{aligned}
& {[A B]_{+}:=\lim _{m \rightarrow \infty} A_{m} B_{m+1}=\left[\begin{array}{cc}
q a, c \\
q^{2} b, q d
\end{array} \mid q\right]_{\infty} \frac{\left(q^{3} b d ; q^{2}\right)_{\infty}}{\left(q a c ; q^{2}\right)_{\infty}}} \\
& {[A B]_{-}:=\lim _{m \rightarrow-\infty} A_{m} B_{m+1}=\left[\begin{array}{c}
1 / q b, 1 / d \\
1 / a, q / c
\end{array} \mid q\right]_{\infty} \frac{\left(q / a c ; q^{2}\right)_{\infty}}{\left(1 / q b d ; q^{2}\right)_{\infty}}}
\end{aligned}
$$

and the following differences:

$$
\begin{aligned}
\nabla A_{k} & =\frac{(1-d / a)\left(1-q^{1+k} b\right)}{(1-q b d)(1-1 / a)} \frac{(a ; q)_{k}\left(q b d ; q^{2}\right)_{k}}{(q d ; q)_{k}\left(q^{3} a b ; q^{2}\right)_{k}} q^{k}, \\
\Delta B_{k} & =\frac{\left(1-c / q^{2} b\right)\left(1-q^{1+k} a\right)}{(1-q a c)\left(1-1 / q^{2} b\right)} \frac{(c ; q)_{k}\left(q^{3} a b ; q^{2}\right)_{k}}{\left(q^{3} b ; q\right)_{k}\left(q^{3} a c ; q^{2}\right)_{k}} q^{k} .
\end{aligned}
$$

By means of the modified Abel lemma on summation by parts, we can manipulate the $\Omega$-series as follows:

$$
\Omega(a, b, c, d) \times \frac{(1-d / a)(1-q b)}{(1-q b d)(1-1 / a)}=\sum_{k} B_{k} \nabla A_{k}=[A B]_{+}-[A B]_{-}+\sum_{k} A_{k} \triangle B_{k} .
$$

Writing explicitly the last sum

$$
\sum_{k} A_{k} \triangle B_{k}=\frac{(1-q a)\left(1-c / q^{2} b\right)}{(1-q a c)\left(1-1 / q^{2} b\right)} \sum_{k}\left[\begin{array}{l}
q^{2} a, c \\
q^{3} b, q d
\end{array} \mid q\right]_{k} \frac{\left(q^{3} b d ; q^{2}\right)_{k}}{\left(q^{3} a c ; q^{2}\right)_{k}} q^{k},
$$

we find the following recurrence relation:

$$
\begin{aligned}
& \Omega(a, b, c, d)=\frac{c}{d} \frac{(1-a)(1-q a)(1-q b d)\left(1-q^{2} b / c\right)}{(1-q b)\left(1-q^{2} b\right)(1-q a c)(1-a / d)} \Omega\left(q^{2} a, q^{2} b, c, d\right) \\
& \quad+\frac{1 / d}{1-a / d}\left[\begin{array}{cc}
a, c \\
q b, q d
\end{array} \mid q\right]_{\infty} \frac{\left(q b d ; q^{2}\right)_{\infty}}{\left(q a c ; q^{2}\right)_{\infty}}+\frac{a}{1-a / d}\left[\begin{array}{c}
1 / b, 1 / d \\
q / a, q / c
\end{array} \mid q\right]_{\infty} \frac{\left(q / a c ; q^{2}\right)_{\infty}}{\left(q / b d ; q^{2}\right)_{\infty}}
\end{aligned}
$$

Iterating this relation $m$ times, we get

$$
\begin{aligned}
& \Omega(a, b, c, d)=\left(\frac{c}{d}\right)^{m}\left[\begin{array}{ccc}
a, q a, q b d, q^{2} b / c \\
q b, q^{2} b, q a c, & a / d
\end{array} \mid q^{2}\right]_{m} \Omega\left(q^{2 m} a, q^{2 m} b, c, d\right) \\
& +\sum_{k=0}^{m-1} \frac{1 / d}{1-q^{2 k} a / d}\left[\begin{array}{cc}
q^{2 k} a, & c \\
q^{1+2 k} b, q d
\end{array} \mid q\right]_{\infty} \frac{\left(q^{1+2 k} b d ; q^{2}\right)_{\infty}}{\left(q^{1+2 k} a c ; q^{2}\right)_{\infty}}\left(\frac{c}{d}\right)^{k}\left[\begin{array}{c}
a, q a, q b d, q^{2} b / c \\
q b, q^{2} b, q a c, a / d
\end{array} \mid q^{2}\right]_{k} \\
& +\sum_{k=0}^{m-1} \frac{q^{2 k} a}{1-q^{2 k} a / d}\left[\begin{array}{c}
q^{-2 k} / b, 1 / d \\
q^{1-2 k} / a, q / c
\end{array} \mid q\right]_{\infty} \frac{\left(q^{1-2 k} / a c ; q^{2}\right)_{\infty}}{\left(q^{1-2 k} / b d ; q^{2}\right)_{\infty}}\left(\frac{c}{d}\right)^{k}\left[\left.\begin{array}{l}
a, q a, q b d, q^{2} b / c \\
q b, q^{2} b, q a c, a / d
\end{array}\right|^{2}\right]_{k} .
\end{aligned}
$$

Simplifying the last equation, we establish the following transformation formula. 


\section{Proposition 8.}

$$
\begin{aligned}
& \Omega(a, b, c, d)=\left(\frac{c}{d}\right)^{m}\left[\begin{array}{l}
a, q a, q b d, q^{2} b / c \\
q b, q^{2} b, q a c, a / d
\end{array} \mid q^{2}\right]_{m} \Omega\left(q^{2 m} a, q^{2 m} b, c, d\right) \\
& +\frac{1 / d}{1-a / d}\left[\begin{array}{cc}
a, c \\
q b, q d
\end{array} \mid q\right]_{\infty} \frac{\left(q b d ; q^{2}\right)_{\infty}}{\left(q a c ; q^{2}\right)_{\infty}} \sum_{k=0}^{m-1} \frac{\left(q^{2} b / c ; q^{2}\right)_{k}}{\left(q^{2} a / d ; q^{2}\right)_{k}}\left(\frac{c}{d}\right)^{k} \\
& +\frac{a}{1-a / d}\left[\begin{array}{l}
1 / b, 1 / d \\
q / a, q / c
\end{array} \mid q\right]_{\infty} \frac{\left(q / a c ; q^{2}\right)_{\infty}}{\left(q / b d ; q^{2}\right)_{\infty}} \sum_{k=0}^{m-1} \frac{\left(q^{2} b / c ; q^{2}\right)_{k}}{\left(q^{2} a / d ; q^{2}\right)_{k}}\left(\frac{a}{b}\right)^{k} .
\end{aligned}
$$

Suppose that $|a / b|<1$ and $|c / d|<1$. When $m \rightarrow \infty$, in order to examine the limit of $\Omega\left(q^{2 m} a, q^{2 m} b, c, d\right)$, we rewrite this series by splitting the bilateral sum into the following three parts:

$$
\begin{aligned}
\left(\frac{c}{d}\right)^{m} \Omega\left(q^{2 m} a, q^{2 m} b, c, d\right) & =\left(\frac{c}{d}\right)^{m} \sum_{k>0}\left[\begin{array}{c}
q^{2 m} a, c \\
q^{1+2 m} b, q d
\end{array} \mid q\right]_{k} \frac{\left(q^{1+2 m} b d ; q^{2}\right)_{k}}{\left(q^{1+2 m} a c ; q^{2}\right)_{k}} q^{k} \\
& +\left(\frac{c}{d}\right)^{m} \sum_{k<-2 m}\left[\begin{array}{cc}
q^{2 m} a, & c \\
q^{1+2 m} b, q d
\end{array} \mid q\right]_{k} \frac{\left(q^{1+2 m} b d ; q^{2}\right)_{k}}{\left(q^{1+2 m} a c ; q^{2}\right)_{k}} q^{k} \\
& +\left(\frac{c}{d}\right)^{m} \sum_{k=-2 m}^{0}\left[\begin{array}{cc}
q^{2 m} a, & c \\
q^{1+2 m} b, q d
\end{array} \mid q\right]_{k} \frac{\left(q^{1+2 m} b d ; q^{2}\right)_{k}}{\left(q^{1+2 m} a c ; q^{2}\right)_{k}} q^{k}
\end{aligned}
$$

It is obvious that the partial series with respect to $k$ displayed in the first line is convergent. Therefore the right member in (3a) tends to zero as $m \rightarrow \infty$. For the partial series displayed in the middle line, performing the replacement $k \rightarrow-2 m-k$ and then applying the relations

$$
\begin{aligned}
& {\left[\begin{array}{cc}
q^{2 m} a, & c \\
q^{1+2 m} b, q d
\end{array} \mid q\right]_{-2 m-k}=\left[\begin{array}{cc}
q b, 1 / d \\
a, & q / c
\end{array} \mid q\right]_{2 m}\left(\frac{q d}{c}\right)^{2 m}\left[\begin{array}{cc}
1 / b, & q^{2 m} / d \\
q / a, & q^{1+2 m} / c
\end{array} \mid q\right]_{k}\left(\frac{q^{2} b d}{a c}\right)^{k},} \\
& \frac{\left(q^{1+2 m} b d ; q^{2}\right)_{-2 m-k}}{\left(q^{1+2 m} a c ; q^{2}\right)_{-2 m-k}}=\left[\left.\begin{array}{l}
q a c, q / a c \\
q b d, q / b d
\end{array}\right|^{2}\right]_{m}\left(\frac{a c}{b d}\right)^{m} \frac{\left(q^{1+2 m} / a c ; q^{2}\right)_{k}}{\left(q^{1+2 m} / b d ; q^{2}\right)_{k}}\left(\frac{a c}{b d}\right)^{k},
\end{aligned}
$$

we can reformulate it as

$$
\left[\begin{array}{cc}
q b, 1 / d \\
a, & q / c
\end{array} \mid q\right]_{2 m}\left[\begin{array}{c}
q a c, q / a c \\
q b d, q / b d
\end{array} \mid q^{2}\right]_{m}\left(\frac{a}{b}\right)^{m} \sum_{k>0}\left[\begin{array}{cc}
1 / b, & q^{2 m} / d \\
q / a, q^{1+2 m} / c
\end{array} \mid q\right]_{k} \frac{\left(q^{1+2 m} / a c ; q^{2}\right)_{k}}{\left(q^{1+2 m} / b d ; q^{2}\right)_{k}} q^{k}
$$

which tends to zero too when $m \rightarrow \infty$.

Finally for the truncated series displayed in (3c), changing the summation index by $k \rightarrow k-m$ and then utilizing the relations

$$
\begin{aligned}
& {\left[\begin{array}{cc}
q^{2 m} a, c \\
q^{1+2 m} b, q d
\end{array} \mid q\right]_{k-m}=\left[\begin{array}{c}
q^{1+m} b, 1 / d \\
q^{m} a, \quad q / c
\end{array} \mid q\right]_{m}\left(\frac{q d}{c}\right)^{m}} \\
& \times\left[\begin{array}{cc}
q^{m} a, & q^{1+m-k} / c \\
q^{1+m} b, & q^{m-k} / d
\end{array} \mid q\right]_{k}\left(\frac{c}{q d}\right)^{k}, \\
& \frac{\left(q^{1+2 m} b d ; q^{2}\right)_{k-m}}{\left(q^{1+2 m} a c ; q^{2}\right)_{k-m}}=\frac{\left(q a c ; q^{2}\right)_{m}}{\left(q b d ; q^{2}\right)_{m}} \frac{\left(q b d ; q^{2}\right)_{k}}{\left(q a c ; q^{2}\right)_{k}},
\end{aligned}
$$

we can express it as

$$
\left[\begin{array}{cc}
q^{1+m} b, 1 / d \\
q^{m} a, & q / c
\end{array} \mid q\right]_{m} \frac{\left(q a c ; q^{2}\right)_{m}}{\left(q b d ; q^{2}\right)_{m}} \sum_{k=-m}^{m}\left[\begin{array}{cc}
q^{m} a, & q^{1+m-k} / c \\
q^{1+m} b, & q^{m-k} / d
\end{array} \mid q\right]_{k} \frac{\left(q b d ; q^{2}\right)_{k}}{\left(q a c ; q^{2}\right)_{k}}\left(\frac{c}{d}\right)^{k} .
$$


When $m \rightarrow \infty$, the limit of the last bilateral sum can be evaluated by Ramanujan's ${ }_{1} \psi_{1}$-sum [5, II-29]

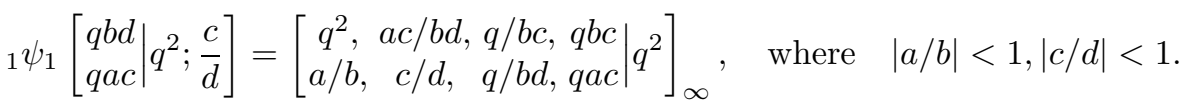

Summing up, we have established the transformation stated in Theorem 1 .

\section{ANOTHER DIFFERENCE PAIR}

Alternatively, define another pair of sequences by

$$
A_{k}=\left[\begin{array}{lc}
q a, q b d / a \\
q b, & q d
\end{array} \mid q\right]_{k} \text { and } B_{k}=\left[\begin{array}{c}
c \\
b d / a
\end{array} \mid q\right]_{k}\left[\begin{array}{l}
q b d \\
q a c
\end{array} \mid q^{2}\right]_{k} .
$$

It is not hard to verify the relations

$$
\begin{aligned}
& {[A B]_{+}:=\lim _{m \rightarrow \infty} A_{m} B_{m+1}=\frac{1}{1-b d / a}\left[\begin{array}{l}
q a, c \\
q b, q d
\end{array} \mid q\right]_{\infty} \frac{\left(q b d ; q^{2}\right)_{\infty}}{\left(q a c ; q^{2}\right)_{\infty}},} \\
& {[A B]_{-}:=\lim _{m \rightarrow-\infty} A_{m} B_{m+1}=\frac{1}{1-a / b d}\left[\begin{array}{c}
1 / b, 1 / d \\
1 / a, q / c
\end{array} \mid\right]_{\infty} \frac{\left(q / a c ; q^{2}\right)_{\infty}}{\left(q / b d ; q^{2}\right)_{\infty}}}
\end{aligned}
$$

and the following differences:

$$
\begin{aligned}
\nabla A_{k} & =\frac{(1-a / b)(1-a / d)}{(1-a)(1-a / b d)}\left[\begin{array}{c}
a, b d / a \\
q b, q d
\end{array} \mid q\right]_{k} q^{k} \\
\Delta B_{k} & =\frac{\left(1-q^{1+k} a\right)(1-a c / b d)}{(1-q a c)(1-a / b d)}\left[\begin{array}{c}
c \\
q b d / a
\end{array} \mid q\right]_{k}\left[\begin{array}{c}
q b d \\
q^{3} a c
\end{array} \mid q^{2}\right]_{k} q^{k} .
\end{aligned}
$$

According to the modified Abel lemma on summation by parts, we can reformulate the $\Omega$-series as follows:

$$
\Omega(a, b, c, d) \times \frac{(1-a / b)(1-a / d)}{(1-a)(1-a / b d)}=\sum_{k} B_{k} \nabla A_{k}=[A B]_{+}-[A B]_{-}+\sum_{k} A_{k} \triangleleft B_{k} .
$$

Observing that the last sum is equal to

$$
\sum_{k} A_{k} \Delta B_{k}=\frac{(1-q a)(1-a c / b d)}{(1-q a c)(1-a / b d)} \sum_{k}\left[\begin{array}{cc}
q^{2} a, c \\
q b, & q d
\end{array} \mid q\right]_{k} \frac{\left(q b d ; q^{2}\right)_{k}}{\left(q^{3} a c ; q^{2}\right)_{k}} q^{k}
$$

we derive the following recurrence relation:

$$
\begin{aligned}
\Omega(a, b, c, d) & =\frac{(1-a)(1-q a)(1-a c / b d)}{(1-a / b)(1-a / d)(1-q a c)} \Omega\left(q^{2} a, b, c, d\right) \\
& +\frac{a}{(1-a / b)(1-a / d)}\left[\begin{array}{c}
1 / b, 1 / d \\
q / a, q / c
\end{array} \mid q\right]_{\infty} \frac{\left(q / a c ; q^{2}\right)_{\infty}}{\left(q / b d ; q^{2}\right)_{\infty}} \\
& -\frac{a / b d}{(1-a / b)(1-a / d)}\left[\begin{array}{cc}
a, c \\
q b, q d
\end{array} \mid q\right]_{\infty} \frac{\left(q b d ; q^{2}\right)_{\infty}}{\left(q a c ; q^{2}\right)_{\infty}}
\end{aligned}
$$

Iterating the last relation $m$-times leads us to the following transformation. 


\section{Proposition 9.}

$$
\begin{aligned}
& \Omega(a, b, c, d)=\left[\begin{array}{ccc}
a, & q a, & a c / b d \\
a / b, a / d, & q a c
\end{array} \mid q^{2}\right]_{m} \Omega\left(q^{2 m} a, b, c, d\right) \\
& +a\left[\begin{array}{c}
1 / b, 1 / d \\
q / a, q / c
\end{array} \mid q\right]_{\infty} \frac{\left(q / a c ; q^{2}\right)_{\infty}}{\left(q / b d ; q^{2}\right)_{\infty}} \sum_{k=0}^{m-1} \frac{\left(a c / b d ; q^{2}\right)_{k} q^{2\left(\begin{array}{c}
k+1 \\
2
\end{array}\right)}}{\left[a / b, a / d ; q^{2}\right]_{k+1}}\left(-\frac{a}{c}\right)^{k} \\
& -\frac{a}{b d}\left[\begin{array}{cc}
a, c \\
q b, q d
\end{array} \mid q\right]_{\infty} \frac{\left(q b d ; q^{2}\right)_{\infty}}{\left(q a c ; q^{2}\right)_{\infty}} \sum_{k=0}^{m-1} \frac{\left(a c / b d ; q^{2}\right)_{k}}{\left[a / b, a / d ; q^{2}\right]_{k+1}} q^{2 k}
\end{aligned}
$$

The asymptotic behavior of $\Omega\left(q^{2 m} a, b, c, d\right)$ as $m \rightarrow \infty$ can be examined analogously. For this purpose, we rewrite the series by splitting it into four parts:

$$
\begin{aligned}
\Omega\left(q^{2 m} a, b, c, d\right) & =\sum_{k \geq 0}\left[\begin{array}{cc}
q^{2 m} a, c \\
q b, & q d
\end{array} \mid q\right]_{k} \frac{\left(q b d ; q^{2}\right)_{k}}{\left(q^{1+2 m} a c ; q^{2}\right)_{k}} q^{k} \\
& +\sum_{k<-2 m}\left[\begin{array}{cc}
q^{2 m} a, & c \\
q b, & q d
\end{array} \mid q\right]_{k} \frac{\left(q b d ; q^{2}\right)_{k}}{\left(q^{1+2 m} a c ; q^{2}\right)_{k}} q^{k} \\
& +\sum_{k=-2 m}^{-m-1}\left[\begin{array}{cc}
q^{2 m} a, c \\
q b, & q d
\end{array} \mid q\right]_{k} \frac{\left(q b d ; q^{2}\right)_{k}}{\left(q^{1+2 m} a c ; q^{2}\right)_{k}} q^{k} \\
& +\sum_{k=-m}^{-1}\left[\begin{array}{cc}
q^{2 m} a, c \\
q b, & q d
\end{array}\right]_{k} \frac{\left(q b d ; q^{2}\right)_{k}}{\left(q^{1+2 m} a c ; q^{2}\right)_{k}} q^{k} .
\end{aligned}
$$

Obviously, when $m \rightarrow \infty$, the partial series displayed in (4a) tends to

$$
\sum_{k \geq 0}\left[\begin{array}{c}
c \\
q b, q d
\end{array} \mid q\right]_{k}\left(q b d ; q^{2}\right)_{k} q^{k}
$$

For the partial series displayed in (4b), performing the replacement $k \rightarrow-k$, we can reformulate it as

$$
\frac{\left(q a c ; q^{2}\right)_{m}}{(a ; q)_{2 m}}\left(-\frac{q^{m-1} a}{c}\right)^{m} \sum_{k>2 m}\left[\begin{array}{c}
1 / b, 1 / d \\
q / c
\end{array} \mid q\right]_{k} \frac{\left(q / a c ; q^{2}\right)_{k-m}}{\left(q / b d ; q^{2}\right)_{k}(q / a ; q)_{k-2 m}} q^{k},
$$

which tends to zero when $m \rightarrow \infty$.

For the finite sum displayed in (4c), it can be restated, under the replacement $k \rightarrow-m-k$, as

$$
\frac{\left(q a c ; q^{2}\right)_{m}}{\left(q^{m} a ; q\right)_{m}} q^{\left(\begin{array}{c}
m+1 \\
2
\end{array}\right) c^{-m}} \sum_{k=1}^{m}\left[\begin{array}{c}
1 / b, 1 / d \\
q / c
\end{array} \mid q\right]_{k+m} \frac{\left(q / a c ; q^{2}\right)_{k} q^{m k-\left(\begin{array}{c}
k \\
2
\end{array}\right)(-a)^{k}}}{\left(q / b d ; q^{2}\right)_{k+m}\left(q^{m-k} a ; q\right)_{k}}
$$

which also tends to zero when $m \rightarrow \infty$.

Finally making the replacement $k \rightarrow-k$, we can express (4d) as

$$
\sum_{k=1}^{m}\left[\begin{array}{c}
1 / b, 1 / d \\
q / c, q^{2 m-k} a
\end{array} \mid q\right]_{k} \frac{\left(q^{1+2 m-2 k} a c ; q^{2}\right)_{k}}{\left(q / b d ; q^{2}\right)_{k}} q^{\left(\begin{array}{c}
k+1 \\
2
\end{array}\right) c^{-k}}
$$

which becomes, as $m \rightarrow \infty$, the series

$$
\sum_{k=1}^{\infty}\left[\begin{array}{c}
1 / b, 1 / d \\
q / c
\end{array} \mid q\right]_{k} \frac{q^{\left(\begin{array}{c}
k+1 \\
2
\end{array}\right) c^{-k}}}{\left(q / b d ; q^{2}\right)_{k}}=\sum_{k=-\infty}^{-1}\left[\begin{array}{c}
c \\
q b, q d
\end{array} \mid q\right]_{k}\left(q b d ; q^{2}\right)_{k} q^{k}
$$


Combining the first and fourth parts together yields a bilateral series. We have therefore derived the nonterminating transformation formula.

\section{Theorem 10.}

$$
\begin{aligned}
& \Omega(a, b, c, d)=\left[\begin{array}{ccc}
a, & q a, a c / b d \\
a / b, a / d, & q a c
\end{array} \mid q^{2}\right]_{\infty} \sum_{k=-\infty}^{\infty}\left[\begin{array}{c}
c \\
q b, q d
\end{array} \mid q\right]_{k}\left(q b d ; q^{2}\right)_{k} q^{k} \\
& +a\left[\begin{array}{c}
1 / b, 1 / d \\
q / a, q / c
\end{array} \mid q\right]_{\infty} \frac{\left(q / a c ; q^{2}\right)_{\infty}}{\left(q / b d ; q^{2}\right)_{\infty}} \sum_{k=0}^{\infty} \frac{\left(a c / b d ; q^{2}\right)_{k} q^{2\left(\begin{array}{c}
k+1 \\
2
\end{array}\right)}}{\left[a / b, a / d ; q^{2}\right]_{k+1}}\left(-\frac{a}{c}\right)^{k} \\
& -\frac{a}{b d}\left[\begin{array}{cc}
a, c \\
q b, q d
\end{array} \mid q\right]_{\infty} \frac{\left(q b d ; q^{2}\right)_{\infty}}{\left(q a c ; q^{2}\right)_{\infty}} \sum_{k=0}^{\infty} \frac{\left(a c / b d ; q^{2}\right)_{k}}{\left[a / b, a / d ; q^{2}\right]_{k+1}} q^{2 k} .
\end{aligned}
$$

Compared with Theorem 1, the last one seems less useful. However, letting $d=a c / b$ in this theorem does result in an unusual identity on the bilateral balancing series

$$
\text { (5) }{ }_{2} \psi_{2}\left[\begin{array}{cc}
a, c \\
q b, q a c / b
\end{array} \mid q ; q\right]=\frac{(1-b)(1-b / a c)}{(1-b / a)(1-b / c)}\left\{\left[\begin{array}{c}
q / b, q b / a c \\
q / a, q / c
\end{array} \mid q\right]_{\infty}-\left[\begin{array}{c}
a, c \\
b, a c / b
\end{array} \mid q\right]_{\infty}\right\} .
$$

Strangely enough, this identity also follows from the telescoping method. In fact, observing the relation

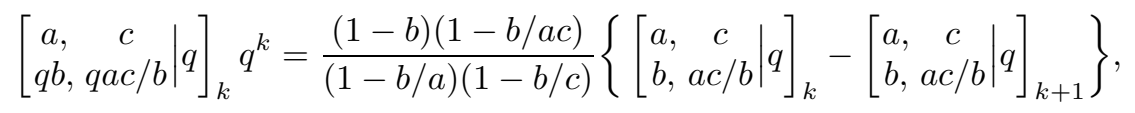

its sum with respect to $k$ from $-\infty$ to $+\infty$ reads as

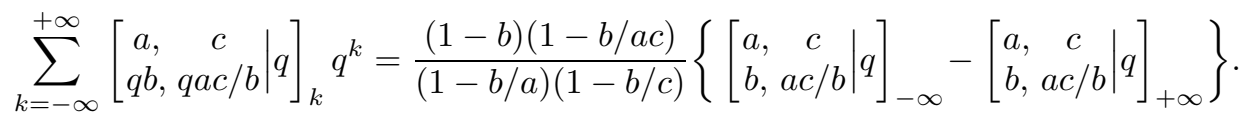

This confirms the identity (5) in view of the equation

$$
\left[\begin{array}{lc}
a, c & c \\
b, a c / b & q
\end{array}\right]_{-\infty}=\left[\begin{array}{cc}
q / b, q b / a c \\
q / a, & q / c
\end{array} \mid q\right]_{\infty}
$$

\section{Review of M. JaCKSON'S IDENTITy OF ${ }_{3} H_{3}$-SERIES}

For the two nonterminating series identities ([8) and ([9]) discovered respectively by Watson and Whipple, there is a common generalization of bilateral series due to M. Jackson [6, Eq. 2.3]:

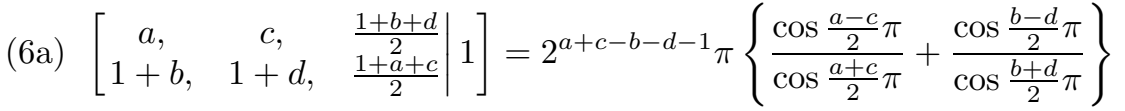

$$
\begin{aligned}
& \times \Gamma\left[\begin{array}{c}
1-a, 1-c, 1+b, 1+d, \frac{2-a-c+b+d}{2} \\
\frac{1-a-c}{2}, \frac{1+b+d}{2}, \frac{2-a+b}{2}, \frac{2-a+d}{2}, \frac{2-c+b}{2}, \frac{2-c+d}{2}
\end{array}\right]
\end{aligned}
$$

provided that $\Re(a+c-b-d)<2$ for convergence. We shall show that this identity follows from Theorem 1 as $q \rightarrow 1^{-}$after having replaced $a, b, c, d$ respectively by their $q$-exponentials $q^{a}, q^{b}, q^{c}, q^{d}$.

By means of Heine's transformation (cf. [5, III-3])

$$
{ }_{2} \phi_{1}\left[\begin{array}{cc}
a, & b \\
& c
\end{array} \mid q ; x\right]=\frac{(a b x / c ; q)_{\infty}}{(x ; q)_{\infty}}{ }_{2} \phi_{1}\left[\begin{array}{cc}
c / a, & c / b \\
& c
\end{array} \mid q ; a b x / c\right]
$$


the equation displayed in Theorem 1 can be reformulated as

(7a) ${ }_{4} \psi_{4}\left[\begin{array}{cccc}a, & c, & \sqrt{q b d}, & -\sqrt{q b d} \\ q b, & q d, & \sqrt{q a c}, & -\sqrt{q a c}\end{array} ; q\right]$

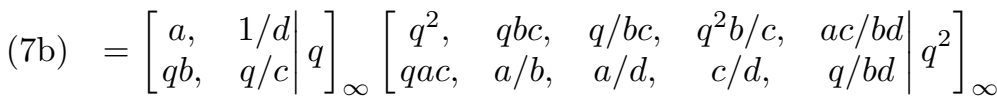

$(7 \mathrm{c})+\frac{1 / d}{1-a / d}\left[\begin{array}{cc}a, & c \\ q b, & q d\end{array} \mid q\right]_{\infty}\left[\begin{array}{c}q b d, q^{2} b / a \\ q a c, c / d\end{array} \mid q^{2}\right]_{\infty}{ }_{2} \phi_{1}\left[\begin{array}{c}a c / b d, a / d \\ q^{2} a / d\end{array} \mid q^{2} ; q^{2} b / a\right]$

$(7 \mathrm{~d})+\frac{a}{1-a / d}\left[\begin{array}{cc}1 / b, & 1 / d \\ q / a, & q / c\end{array}\right]_{\infty}\left[\begin{array}{c}q / a c, q^{2} d / c \mid \\ q / b d, a / b\end{array} \mid q^{2}\right]_{\infty}{ }_{2} \phi_{1}\left[\begin{array}{c}a c / b d, a / d \mid \\ q^{2} a / d\end{array} \mid q^{2} ; q^{2} d / c\right]$.

This expression permits us to realize the limiting process as $q \rightarrow 1^{-}$. Recall the $q$-Gamma function (cf. [5, I-35])

$$
\Gamma_{q}(x)=(1-q)^{1-x} \frac{(q ; q)_{\infty}}{\left(q^{x} ; q\right)_{\infty}} \quad \text { with } \quad \lim _{q \rightarrow 1^{-}} \Gamma_{q}(x)=\Gamma(x)
$$

Replacing $a, b, c, d$ by their $q$-exponentials $q^{a}, q^{b}, q^{c}, q^{d}$ in the last transformation and then letting $q \rightarrow 1^{-}$, we may evaluate separately the expressions from (7a) to (7d) as follows. First for the ${ }_{4} \psi_{4}$-series in (7ad), it is trivial to see that the series becomes

$$
{ }_{3} H_{3}\left[\begin{array}{ccc}
a, & c, & \frac{1+b+d}{2} \\
1+b, & 1+d, & \frac{1+a+c}{2}
\end{array} \mid 1\right]
$$

which is convergent under the condition $\Re(a+c-b-d)<2$. Under the same condition, the limits of both ${ }_{2} \phi_{1}$-series in (7c) and (7d) can be evaluated, through the Gauss summation theorem (cf. [2, §1.3]), as follows:

$$
{ }_{2} F_{1}\left[\begin{array}{c}
\frac{a+c-b-d}{2}, \frac{a-d}{2} \mid \\
1+\frac{a-d}{2} \mid
\end{array}\right]=\Gamma\left[\begin{array}{c}
\frac{2+a-d}{2}, \frac{2-a-c+b+d}{2} \\
\frac{2+b-c}{2}
\end{array}\right] .
$$

For the $\Gamma$-function, keeping in mind the reciprocal relations

$$
\Gamma(x) \Gamma(1-x)=\frac{\pi}{\sin \pi x} \quad \text { and } \quad \Gamma\left(\frac{1}{2}+x\right) \Gamma\left(\frac{1}{2}-x\right)=\frac{\pi}{\cos \pi x}
$$

as well as Legendre's duplication formula

$$
\Gamma(x) \Gamma\left(\frac{1}{2}+x\right)=\frac{\sqrt{\pi}}{2^{2 x-1}} \Gamma(2 x),
$$


we can further derive the following expressions:

$$
\begin{aligned}
& (17 \mathrm{~b}) \Rightarrow \Gamma\left[\begin{array}{l}
\frac{1+b}{2}, \frac{2+b}{2}, \frac{1-c}{2}, \frac{2-c}{2}, \frac{1+a+c}{2}, \frac{1-b-d}{2}, \frac{a-b}{2}, \frac{a-d}{2}, \frac{c-d}{2} \\
\frac{a}{2}, \frac{1+a}{2}, \frac{-d}{2}, \frac{1-d}{2}, \frac{1+b+c}{2}, \frac{1-b-c}{2}, \frac{2+b-c}{2}, \frac{a+c-b-d}{2}
\end{array}\right] \\
& =\Gamma\left[\begin{array}{c}
1-a, 1-c, 1+b, 1+d, \frac{2-a-c+b+d}{2} \\
\frac{1-a-c}{2}, \frac{1+b+d}{2}, \frac{2-a+b}{2}, \frac{2-a+d}{2}, \frac{2-c+b}{2}, \frac{2-c+d}{2}
\end{array}\right] \\
& \times \quad \frac{\pi}{2^{2-a-c+b+d}} \frac{\sin a \pi \sin d \pi \sin \frac{b+d-a-c}{2} \pi \cos \frac{b+c}{2} \pi}{\sin \frac{a-b}{2} \pi \sin \frac{a-d}{2} \pi \sin \frac{c-d}{2} \pi \cos \frac{a+c}{2} \pi \cos \frac{b+d}{2} \pi} ; \\
& \left(\underline{7 c)} \Rightarrow \Gamma\left[\begin{array}{c}
\frac{1+b}{2}, \frac{2+b}{2}, \frac{1+d}{2}, \frac{2+d}{2}, \frac{1+a+c}{2}, \frac{a-d}{2}, \frac{c-d}{2}, \frac{2-a-c+b+d}{2} \\
\frac{a}{2}, \frac{1+a}{2}, \frac{c}{2}, \frac{1+c}{2}, \frac{1+b+d}{2}, \frac{2-a+b}{2}, \frac{2-c+b}{2}
\end{array}\right]\right. \\
& =\Gamma\left[\begin{array}{c}
1-a, 1-c, 1+b, 1+d, \frac{2-a-c+b+d}{2} \\
\frac{1-a-c}{2}, \frac{1+b+d}{2}, \frac{2-a+b}{2}, \frac{2-a+d}{2}, \frac{2-c+b}{2}, \frac{2-c+d}{2}
\end{array}\right] \\
& \times \frac{\pi}{2^{2-a-c+b+d}} \frac{\sin a \pi \sin c \pi}{\sin \frac{a-d}{2} \pi \sin \frac{c-d}{2} \pi \cos \frac{a+c}{2} \pi} \text {; } \\
& \text { (7d) } \Rightarrow \Gamma\left[\begin{array}{c}
\frac{1-a}{2}, \frac{2-a}{2}, \frac{1-c}{2}, \frac{2-c}{2}, \frac{1-b-d}{2}, \frac{a-b}{2}, \frac{a-d}{2}, \frac{2-a-c+b+d}{2} \\
\frac{-b}{2}, \frac{1-b}{2}, \frac{-d}{2}, \frac{1-d}{2}, \frac{1-a-c}{2}, \frac{2-c+b}{2}, \frac{2-c+d}{2}
\end{array}\right] \\
& =\Gamma\left[\begin{array}{c}
1-a, 1-c, 1+b, 1+d, \frac{2-a-c+b+d}{2} \\
\frac{1-a-c}{2}, \frac{1+b+d}{2}, \frac{2-a+b}{2}, \frac{2-a+d}{2}, \frac{2-c+b}{2}, \frac{2-c+d}{2}
\end{array}\right] \\
& \times \frac{\pi}{2^{2-a-c+b+d}} \frac{\sin b \pi \sin d \pi}{\sin \frac{a-b}{2} \pi \sin \frac{a-d}{2} \pi \cos \frac{b+d}{2} \pi} .
\end{aligned}
$$

Now, we just need to prove that the sum of the last three trigonometric fractions equals the trigonometric expression displayed in (6a), i.e.

$$
\begin{aligned}
2 \frac{\cos \frac{a-c}{2} \pi}{\cos \frac{a+c}{2} \pi} & +2 \frac{\cos \frac{b-d}{2} \pi}{\cos \frac{b+d}{2} \pi}=\frac{\sin a \pi \sin d \pi \sin \frac{b+d-a-c}{2} \pi \cos \frac{b+c}{2} \pi}{\sin \frac{a-b}{2} \pi \sin \frac{a-d}{2} \pi \sin \frac{c-d}{2} \pi \cos \frac{a+c}{2} \pi \cos \frac{b+d}{2} \pi} \\
& +\frac{\sin a \pi \sin c \pi}{\sin \frac{a-d}{2} \pi \sin \frac{c-d}{2} \pi \cos \frac{a+c}{2} \pi}+\frac{\sin b \pi \sin d \pi}{\sin \frac{a-b}{2} \pi \sin \frac{a-d}{2} \pi \cos \frac{b+d}{2} \pi} .
\end{aligned}
$$

Multiplying $a, b, c, d$ by $2 / \pi$, we can equivalently rewrite the last equation as follows:

$$
\begin{aligned}
2 \sin (a-b) \sin (a-d) \sin (c-d) & \times\{\cos (a-c) \cos (b+d)+\cos (a+c) \cos (b-d)\} \\
& =\sin 2 a \sin 2 d \sin (b+d-a-c) \cos (b+c) \\
& +\sin 2 a \sin 2 c \cos (b+d) \sin (a-b) \\
& +\sin 2 b \sin 2 d \cos (a+c) \sin (c-d) .
\end{aligned}
$$

This will be confirmed by utilizing the following elementary trigonometric formulae:

$$
\begin{aligned}
& \sin 2 a=2 \sin a \cos a, \\
& \sin (a+b)=\sin a \cos b+\sin b \cos a, \\
& \cos (a+b)=\cos a \cos b-\sin a \sin b .
\end{aligned}
$$


Firstly, according to (10b), we have

$$
\sin (b+d-a-c)=\sin (b-a) \cos (d-c)+\sin (d-c) \cos (b-a),
$$

which splits the expression displayed in (9b) into two parts:

$$
\begin{aligned}
(9 \mathrm{~b}) & =\sin 2 a \sin 2 d \sin (b-a) \cos (d-c) \cos (b+c) \\
& +\sin 2 a \sin 2 d \sin (d-c) \cos (b-a) \cos (b+c) .
\end{aligned}
$$

Then combining (11a) with (9c) and (11b) with (9d) respectively, we get the following two expressions:

$$
\begin{aligned}
& (9 \mathrm{c})+(11 \mathrm{a})=\sin 2 a \sin (a-b)\{\sin 2 c \cos (b+d)-\sin 2 d \cos (c-d) \cos (b+c)\}, \\
& (9 \mathrm{~d})+(11 \mathrm{~b})=\sin 2 d \sin (c-d)\{\sin 2 b \cos (a+c)-\sin 2 a \cos (a-b) \cos (b+c)\} .
\end{aligned}
$$

By means of (10a) and (10c), expanding the expression in the braces $\{\cdots\}$ of (12a) and then canceling the common terms, we may simplify it into

$$
\begin{aligned}
& 2\left\{\begin{array}{c}
\sin c \cos b \cos c \cos ^{3} d-\sin d \cos b \cos ^{2} c \cos ^{2} d \\
+\sin b \sin ^{2} c \sin ^{2} d \cos d-\sin b \sin c \sin ^{3} d \cos c
\end{array}\right\} \\
= & 2 \sin (c-d)\left\{\sin b \sin c \sin ^{2} d+\cos b \cos c \cos ^{2} d\right\},
\end{aligned}
$$

which leads us further to the following equality:

$$
\text { (9c) }+ \text { (11a) }=4 \sin (a-b) \sin (c-d) \sin a \cos a\left\{\sin b \sin c \sin ^{2} d+\cos b \cos c \cos ^{2} d\right\} \text {. }
$$

Observing that (12b) can be deduced from (12a) by interchanging the parameters $a \rightleftharpoons d$ and $b \rightleftharpoons c$, we have from the last expression

$$
(9 \mathrm{~d})+(11 \mathrm{~b})=4 \sin (b-a) \sin (c-d) \sin d \cos d\left\{\sin ^{2} a \sin b \sin c+\cos ^{2} a \cos b \cos c\right\} \text {. }
$$

From these two expressions just displayed, we find that

$$
\begin{aligned}
& (9 \mathrm{~b})+(9 \mathrm{c})+(\underline{9 \mathrm{~d}}=(11 \mathrm{a})+(11 \mathrm{~b})+(9 \mathrm{c})+(9 \mathrm{~d}) \\
= & 4 \sin (a-b) \sin (c-d)\left\{\begin{array}{l}
\sin a \cos a \cos b \cos c \cos ^{2} d-\sin d \cos ^{2} a \cos b \cos c \cos d \\
+\sin a \sin b \sin c \sin ^{2} d \cos a-\sin ^{2} a \sin b \sin c \sin d \cos d
\end{array}\right\} \\
= & 4 \sin (a-b) \sin (c-d) \sin (a-d)\{\cos a \cos b \cos c \cos d-\sin a \sin b \sin c \sin d\} .
\end{aligned}
$$

Finally, we can easily verify, by $(10 \mathrm{c})$, the following equation:

$$
\begin{aligned}
& 2\{\cos a \cos b \cos c \cos d-\sin a \sin b \sin c \sin d\} \\
= & \cos (a-c) \cos (b+d)+\cos (a+c) \cos (b-d) .
\end{aligned}
$$

This completes the proof of the trigonometric identity stated in $9 \mathrm{a}, 9 \mathrm{~b}-9 \mathrm{c}$ ) and consequently the identity (6a) due to M. Jackson (1949).

\section{REFERENCES}

[1] G. E. Andrews, On q-analogues of the Watson and Whipple summations, SIAM J. Math. Anal. 7:3 (1976), 332-336. MR0399529 (53:3373)

[2] W. N. Bailey, Generalized Hypergeometric Series, Cambridge University Press, Cambridge, 1935. MR0185155 (32:2625)

[3] W. Chu, Bailey's very well-poised $6 \psi_{6}$-series identity, J. Combin. Theory Ser. A 113:6 (2006), 966-979. MR2244127 (2007g:33015)

[4] W. Chu, Abel's lemma on summation by parts and basic hypergeometric series, Adv. in Appl. Math. 39:4 (2007), 490-514. MR2356433 (2009c:33034)

[5] G. Gasper and M. Rahman, Basic Hypergeometric Series (2nd ed.), Cambridge University Press, Cambridge, 2004. MR2128719(2006d:33028) 
[6] M. Jackson, A generalization of the theorems of Watson and Whipple on the sum of the series ${ }_{3} F_{2}$, J. London Math. Soc. 24 (1949), 238-240. MR0032049(11:246c)

[7] V. K. Jain, Some transformations of basic hypergeometric functions. II, SIAM J. Math. Anal. 12:6 (1981), 957-961. MR0657137 (83d:33004b)

[8] G. N. Watson, A note on generalized hypergeometric series, Proc. London Math. Soc. (2), 23 (1925), xiii-xv.

[9] F. J. W. Whipple, A group of generalized hypergeometric series: Relations between 120 allied series of the type $F[a, b, c ; d, e]$, Proc. London Math. Soc. (2), 23 (1925), 104-114.

Institute of Combinatorial Mathematics, Hangzhou Normal University, Hangzhou 310036, People's Republic of China

Current address: Dipartimento di Matematica, Università del Salento, Via Provinciale LecceArnesano, P.O. Box 193, Lecce 73100, Italy

E-mail address: chu.wenchang@unisalento.it

College of Mathematics and Physics, Nanjing University of Information Science and Technology, Nanjing 210044, People's Republic of China

E-mail address: wang.chenying@163.com 